\title{
QUANTITATIVE INTERPRETATION OF LUMBAR MUSCLE MYOELECTRIC SIGNALS DURING RAPID CYCLIC ATTEMPTED TRUNK FLEXIONS AND EXTENSIONS
}

\author{
D. G. Thelen, A. B. Schultz* and J. A. Ashton-Miller \\ Department of Mechanical Engineering and Applied Mechanics, University of Michigan Ann Arbor, \\ MI 48109-2125, U.S.A.
}

\begin{abstract}
The quantitative relationship between lumbar myoelectric signals (MES) and rapidly varying isometric trunk muscle forces was investigated. Ten young adult males were asked to cycle harmonically between attempted trunk flexion and attempted trunk extension in an upright position at rates of $0.33,0.67$ and $1.0 \mathrm{~Hz}$ to peak efforts of 20,40 and $60 \%$ of maximum voluntary exertion levels. The forces voluntarily exerted against a load cell were measured and used along with acquired kinematic data to calculate the time course of the net sagittal moment at the level of the third lumbar vertebra during task performances. A 22 muscle double linear programming biomechanical model was used to predict the lumbar trunk muscle contraction forces from the calculated moments. Rectified and bidirectionally low-pass filtered myoelectric activities were acquired at the $\mathrm{L} 3$ level from four abdominal muscles and four back muscles. The processed MES were found to be well correlated $(r>0.90)$ with predicted muscle forces when the MES were time-shifted to account for electromechanical delay as well as the dynamic phase shift between muscle electrical activity and contraction force. Mean time shifts that maximized the linear MES-force relationship ranged from 111 to $218 \mathrm{~ms}$, were greater for the trunk extensors than the trunk flexors and generally exhibited lateral symmetry. The corresponding approximate phase angles averaged $20^{\circ}$ at the slowest rate and $50^{\circ}$ at the fastest rate. MES-force phase angles decreased as effort level was increased indicating that the dynamic MES-force relationship is nonlinear. These results illustrate the importance of accounting for the phasc lag between muscle electrical activity and force when using MES to quantify muscle loads during rapidly varying exertions.
\end{abstract}

\section{INTRODUCTION}

Quantification of internal trunk loads during physical task performances is useful to investigate the association of those loads with low back pain and can lead to methods of reducing the prevalence of that condition. During heavy physical tasks, trunk muscle contraction forces largely determine the compression and shear loads imposed on the spine. Measurement of myoelectric signals (MES) during physical task performances provides an indirect means of accessing muscle loads. The quantitative relationship between lumbar MES and the moments about the lumbar spine have been studied during static weight holding (Seroussi and Pope, 1987) and quasi-static attempted exertions in the sagittal plane (Vink et al., 1988; Stokes et al., 1987, 1989). In addition, MES from abdominal and back muscles have been used to validate optimization-based muscle force prediction models (Schultz et al., 1983, 1987; Zetterberg et al., 1987, for example). In the latter studies, good agreement between predicted lumbar muscle forces and MES were found for low-tomoderate effort level tasks (Schultz et al., 1983). However, the linear correlation between predicted muscle forces and MES was weaker for tasks involving asymmetric loadings of the trunk and heavy exertions (Schultz et al., 1987).

Received in final form 7 June 1993.

*Author to whom correspondence should be addressed.
Based on the results of these studies, it is possible to use lumbar MES to estimate trunk loading during the performance of static tasks with a reasonable degree of accuracy. For this purpose, it is first nccessary to calibrate either the MES-force or MES-moment relationship for an individual subject from data taken during a series of static tasks. Then, in other static tasks performed by this subject, the trunk loads can be predicted from myoelectric measurements alone.

Many physical tasks result in dynamic loadings of the trunk. Although it is desirable to use myoelectric signals to estimate rapidly varying muscle forces during such tasks, less is known about the relationship hetween a muscle's myoelectric signal and its timevarying contraction force. McGill and Norman (1986) used lumbar MES from six muscles and a priori assumptions about the MES-force relationship to divide a dynamic sagittal moment into its muscular contributions. However, they concluded that more work needed to be done to find a suitable method of interpreting the myoelectric activities in terms of dynamic musclc contraction force levels.

The purpose of the present study was to investigate the relationship between lumbar MES and isometric muscle contraction forces in rapidly varying efforts. Studying this relationship in isometric tasks removes major methodological complications: for example, muscle length-tension and force-velocity effects become small. Specifically, the quantitative relationship between MES and force was studied during cyclic 
bidirectional exertions in trunk flexion and extension. The effects of cyclic rate and effort level on the gain and phase relationships between MES and muscle tension were determined.

\section{METHODS}

\section{Subjects and tasks}

Ten healthy male adult volunteers served as subjects. Their ages ranged from 21 to 27 years with a mean of 23.5 years; heights ranged from 168 to $191 \mathrm{~cm}$ with a mean of $177 \mathrm{~cm}$; weights ranged from 555 to $800 \mathrm{~N}$ with a mean of $720 \mathrm{~N}$.

All tasks were performed in a test frame designed to allow the subject to attempt trunk flexions and extensions of various magnitudes while he was restrained in an upright position such that minimal motion occurred (Fig. 1). The subject's pelvis and legs were strapped to a support board to stabilize his lower body segments. A strap fastened around the chest just under the arms was connected to a load cell. All tasks were performed with the subject's arms folded comfortably in front of him.

The subjects were asked to perform 11 quasi-static and 9 cyclic attempted exertion tasks with effort levels displayed to them on an oscilloscope. During static tasks, the subjects were asked to attempt trunk flexion or trunk extension to a specified magnitude. During cyclic tasks, the subjects were asked to cycle harmonically between attempted trunk flexion and attempted trunk extension at a given rate and to specified peak effort levels. The subjects were asked to develop the requested attempted trunk flexions and extensions primarily with their trunk muscles and to minimize head, shoulder and arm motions during all tasks.

Static tasks were performed for $2 \mathrm{~s}$ intervals with at least $1 \mathrm{~min}$ rest between tasks. Three static calibration tasks were performed first: stand relaxed, exert maximum effort in attempted trunk flexion, and exert maximum effort in attempted trunk extension. Exertion levels in all other tasks were prescribed based upon the forces developed in these maximum effort tasks. The remaining eight static tasks consisted of attempted trunk flexions and attempted trunk extensions to peak force magnitudes of $10,20,40$ and $60 \%$ of the maximum effort magnitudes.

During cyclic tasks, the attempts were performed at rates guided by a metronomc. Cyclic tasks were performed at 20,40 and 60 counts per minute (cpm). The $60 \mathrm{cpm}$ rate was slightly below the fastest rate at which subjects could alternate between attempted flexion and extension up to a $60 \%$ effort level. At each rate, the subject performed tasks to peak flexion and extension exertion levels of approximately 20,40 and $60 \%$ of maximum effort magnitudes. Three cycles of data were acquired for each cyclic task.

\section{Data measurement and analysis}

During task performance, three types of data were recorded: load cell, kinematic, and MES data. A triggering system was used to synchronize all data

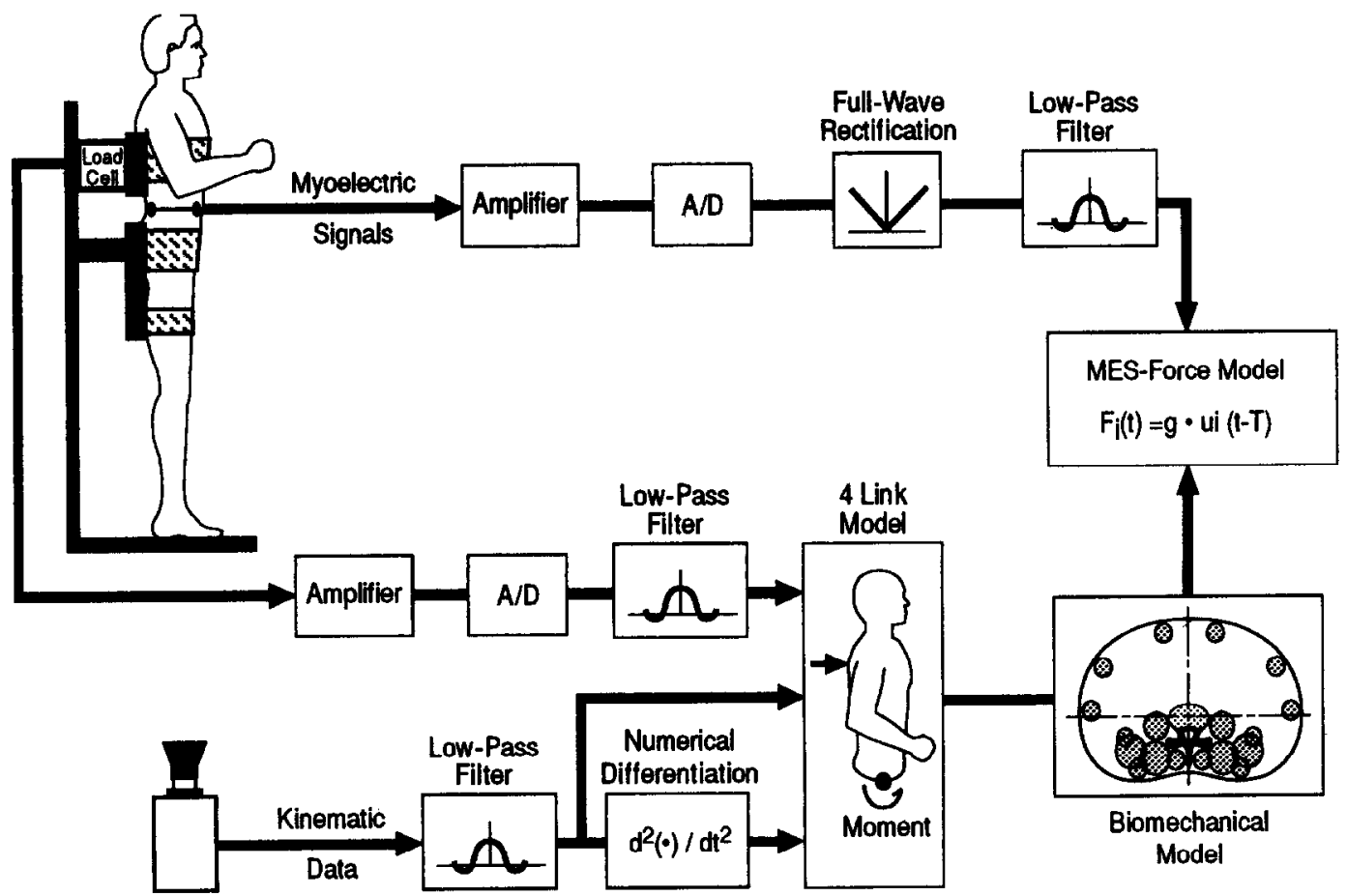

Fig. 1. Schematic diagram of experimental test setup and subsequent data analysis. 
recorded. Load cell data were amplified and digitally acquired at $300 \mathrm{~Hz}$.

Kinematic data were recorded at $300 \mathrm{~Hz}$ using a Watsmart infrared marker optoelectronic camera system. Markers were placed on the right side of the trunk lateral to the center of the vertebral bodies at the L3, L1, T10 and C7 levels. Additional markers were placed on the shoulder, on the arm at $75 \%$ of the distance from the shoulder to the elbow, over the ear and on the load cell. All kinematic data were digitally acquired at $300 \mathrm{~Hz}$.

Load cell and kinematic data were digitally lowpass-filtered using a second-order Butterworth filter with three forward and backward passes to remove phase shift and a cutoff frequency of $4 \mathrm{~Hz}$. The load cell and kinematic data were then resampled at $100 \mathrm{~Hz}$.

The moment in the sagittal plane at the L3 trunk level was calculated using a four-rigid-link model to analyze upper body dynamics. The abdominal trunk segment superior to $\mathrm{L} 3$ was represented by Link 1 , the thorax was represented by Link 2 , the neck and head by Link 3 and the arms by Link 4 . The net moment at the L3 level of the trunk due to external loading, inertial forces, and body segment weights was calculated.

The angle of each link with respect to the vertical at each time step was calculated using two markers located on the associated body segments. The angle and displacement data from the marker at $\mathrm{L} 3$ were numerically double differentiated using a five-point differentiation scheme to estimate velocities and accelerations. The link angular position, velocity and acceleration data, the $\mathrm{L} 3$ linear position and acceleration data, and the load cell data were used along with the four-rigid-link model and anthropometric data (McConville et al., 1980) to calculate the net L3 flexion-extension moment at each time step.

Trunk muscle contraction forces were predicted from the calculated moment using a 22 muscle biomechanical model of the $\mathrm{L} 3$ cross section. Muscle cross-sectional areas and centroid locations were based on the anatomical drawings of Eycleshymer and Schoemaker (1911) and taken from Schultz (1990). The double linear programming biomechanical model is described in detail by Bean et al. (1988). Briefly, this model uses two linear programs to predict which trunk muscle contraction forces are used to produce the calculated moments. In the first program, the objective was to minimize the maximum muscle contraction intensity necessary to develop the moment. In the second program, the objective was to minimizc the compression on the spine subject to the intensity bound found in the first program. Use of these objective functions implies the assumption of minimal antagonistic muscle contractions in sagittal tasks.

MES were recorded from four left and four rightsided bipolar surface electrodes. Two pairs of silver electrodes were placed over the longissimus (ESL) and iliocostalis (ESI) columns of the crector spinae muscle at the $\mathrm{L} 3$ level, 3 and $6 \mathrm{~cm}$ from the midline. A third electrode pair was placed over the rectus abdominis (RA) muscles, $3 \mathrm{~cm}$ lateral to the midline at the level of umbilicus. The fourth electrode pair was placed over the medial oblique external (MOE) muscles, $3 \mathrm{~cm}$ superior and medial to the anterior iliac spine. The electrodes were filled with electrode jelly and taped to the skin. The longitudinal axis of each electrode was aligned approximately with the direction of the underlying muscle fibers.

Raw MES were differentially preamplified with a gain of 667. Signals were further amplified to volt levels and digitized at $900 \mathrm{~Hz}$ per channel using a 12 bit analog-to-digital converter. All MES were displayed on an oscilloscope and visually inspected for artifacts during task performances. All myoelectric signals were then full wave rectified and digitally lowpass-filtered using a second-order Butterworth filter with three forward and backward passes and a cutoff frequency of $4 \mathrm{~Hz}$. The rectified and filtered MES were then resampled at $100 \mathrm{~Hz}$.

For the static tasks, mean biomechanical modelpredicted muscle forces and mean full-wave rectified myoelectric signals were calculated over the $2 \mathrm{~s}$ recording interval for each of the eight muscles monitored. A linear model relating the static trunk muscle forces to the mean rectified activities was assumed in the form

$$
\bar{F}_{i j}=g_{\mathrm{s}} \bar{u}_{i j}+e_{i j},
$$

where $\bar{F}_{i j}$ and $\bar{u}_{i j}$ are the mean biomechanical model predicted muscle contraction force and mean fullwave rectified myoelectric signals for muscle $i$ in task $j$, and $g_{\mathbf{s}}$ is the static MES-force gain parameter. The error, $e_{i j}$, is the difference between the biomechanical model and MES-predicted force for muscle $i$ in task $j$. Gain parameters that minimized the summation of errors squared for each muscle over nine static tasks (stand relaxed; $10,20,40$ and $60 \%$ of maximum efforts in flexion and extension) were calculated.

The cyclic tasks studied involved alternating flexor and extensor muscle activities. Coactivation of agonistic muscles was evaluated by calculating the linear correlation between pairs of rectified and filtered MES. Perfect coactivation, or constant load sharing, between any two muscles would result in a correlation coefficient equal to 1.0 .

For the cyclic isometric attempted exertion tasks, a trunk muscle myoelectric activity-force relationship was assumed in the form

$$
F_{i}(t)=g_{\mathrm{c}} u_{i}(t-T)+e_{i}(t)
$$

where $F_{i}(t)$ is the biomechanical model-predicted force for muscle $i$ at time $t, u_{i}(t)$ is the full-waverectified and low-pass-filtered myoelectric signal at time $t$, and $g_{\mathrm{c}}$ and $T$ are parameters to be fit to the data. For each cyclic task, the time shift $T$ was selected as that value which maximized the linear cross-correlation between the predicted muscle force and timeshiftcd rectified and filtered MES. Least-squares 
regression was then performed on the force and time-shifted processed MES data to calculate the cyclic MES-force gain parameter, $g_{c}$, that minimized the summation of errors squared. The analysis was performed on two cycles of data for each task. A halfcycle of force and MES data were discarded at either end of the recorded time interval in order to eliminate startup and end transients from the low-pass filter.

The goodness-of-fit of all MES-force models was evaluated by a coefficient of variability (CV) which was defined as the root-mean-square error normalized by the root-mean-square predicted muscle force. For static tasks, the root-mean-square error and rootmean-squared biomechanical model-predicted muscle forces was calculated with data from the nine static tasks of $60 \%$ effort and below. For each cyclic at- tempted flexion-extension task, the root-mean-square errors and root-mean-square muscle forces were calculated over two cycles of data.

\section{RESULTS}

Sample correlation plots between biomechanical model-predicted forces and mean rectified MES for two abdominal muscles and two back muscles of one subject demonstrate the linear MES-force relationship found over the series of static exertions (Fig. 2). The average error between the optimization-based and MES-predicted muscle forces ranged from 22 to $24 \%$ over the static attempted flexions and extensions (Table 1).
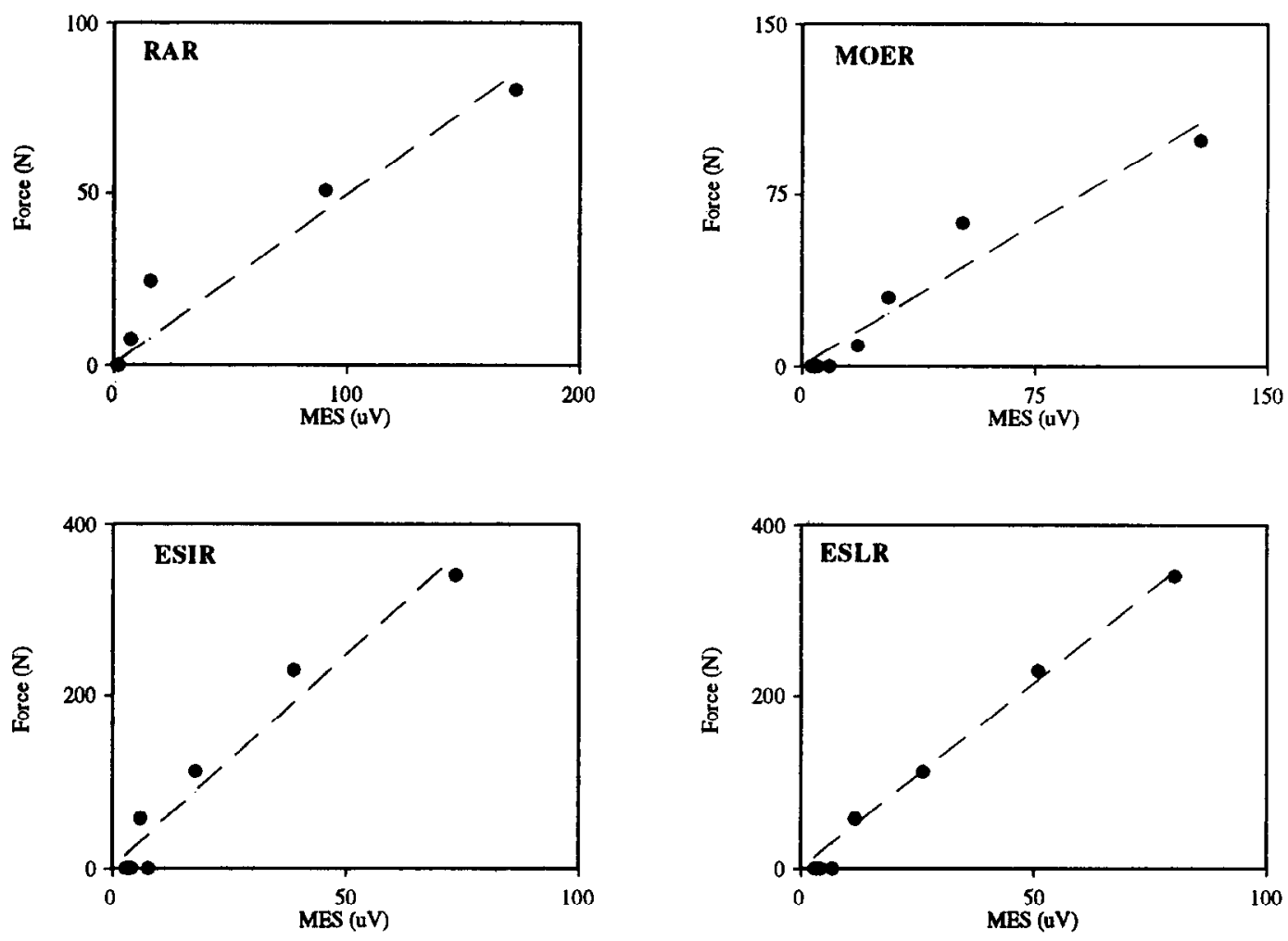

Fig. 2. Sample plots of the mean predicted force versus mean rectified MES during the static attempted trunk flexions and extensions demonstrate a nearly linear relationship.

Table 1. Summary of the static task MES-force gains when using regression through the origin. Also given are the errors between biomechanical model and MES-predicted muscle force as expressed by the coefficient of variability

\begin{tabular}{|c|c|c|c|c|c|c|c|c|}
\hline & \multicolumn{2}{|c|}{ RA } & \multicolumn{2}{|c|}{ MOE } & \multicolumn{2}{|c|}{ ESL } & \multicolumn{2}{|c|}{ ESI } \\
\hline & Mean & SD & Mean & SD & Mean & SD & Mean & SD \\
\hline$\underset{\mathrm{CV}}{g_{\mathrm{s}}(\mathrm{N} / \mu \mathrm{V})}$ & $\begin{array}{l}1.53 \\
0.23\end{array}$ & $\begin{array}{l}(0.82) \\
(0.10)\end{array}$ & $\begin{array}{l}1.64 \\
0.22\end{array}$ & $\begin{array}{l}(0.76) \\
(0.07)\end{array}$ & $\begin{array}{l}4.61 \\
0.24\end{array}$ & $\begin{array}{l}(1.53) \\
(0.09)\end{array}$ & $\begin{array}{l}3.13 \\
0.23\end{array}$ & $\begin{array}{l}(0.81) \\
(0.13)\end{array}$ \\
\hline
\end{tabular}

Note: RA, rectus abdominis; MOE, medial oblique external; ESL, erector spinae longissimus; ESI, erector spinae iliocostalis. 
The time history of the $\mathrm{L} 3$ moment developed during a cyclic task performed at $40 \mathrm{cpm}$ and to $60 \%$ effort level demonstrates that the moment due to the force voluntarily exerted against the load cell was the major contributor to the net moment (Fig. 3). Inertial effects generally contributed less than $15 \%$ to the net moment. For all cyclic attempted exertions tasks, distinct bursts of abdominal and back muscle activities were observed during attempted trunk flexion and extension, respectively (Fig. 4). Rectified and filtered MES from the ESL and ESI muscles were highly cross-correlated with the mean correlation coefficient $(r)$ exceeding 0.95 for all cyclic tasks. The processed MES from the RA and MOE muscle cross-correlated with $r$ greater than 0.83 for the low effort level tasks and $r$ greater than 0.91 for the highest effort level tasks (Table 2).

Minimal overlap of the abdominal and back muscle bursts of myoelectric activity was observed although the abdominal activity between bursts increased as the task rate was increased (Figure 5). A phase difference between the rectified and low-pass filtered MES and predicted muscle forces was evident for all muscles (Figs. 6 and 7). The mean time shifts that maximized the linear correlation between rectified and filtered MESs and biomechanical model-predicted muscle forces ranged from 111 to $218 \mathrm{~ms}$ across the nine cyclic tasks (Table 3). The mean time shifts at each rate and
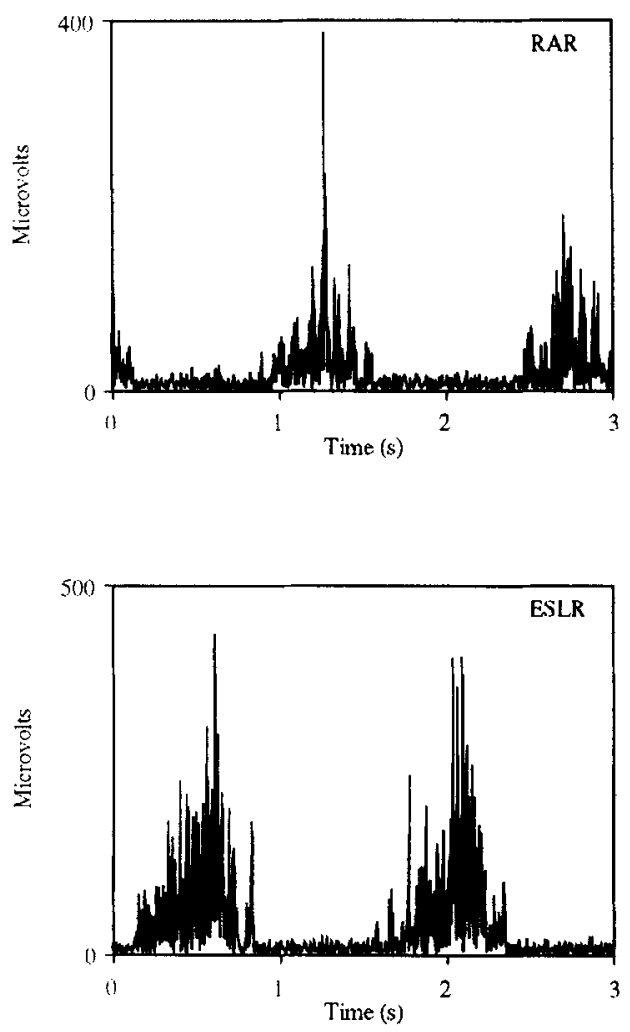

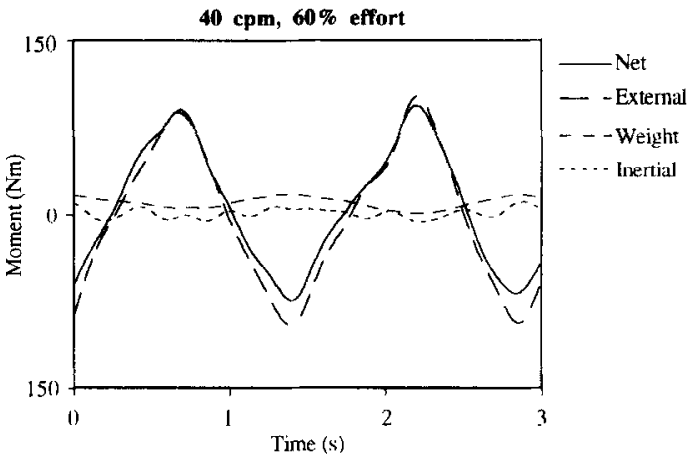

Fig. 3. The moments at the L3 level due to forces voluntarily exerted against a load cell, inertial forces due to upper body segment motions and weight forces due to upper body segment eccentricity during a cyclic isometric task. The force voluntarily exerted against the load cell was the major contributor to the net moment. A positive moment represents attempted extension and a negative moment represents attempted flexion.

effort level were greater for the trunk extensors than the trunk flexors, particularly for the back muscle electrode located $3 \mathrm{~cm}$ from the midline of the body. The MES-force time shifts decreased as the task rate and effort level were increased. For example, the mean time shifts at the $20 \mathrm{cpm}$ and $20 \%$ effort level were $202 \mathrm{~ms}$ for the RA, $180 \mathrm{~ms}$ for the MOE, $218 \mathrm{~ms}$ for
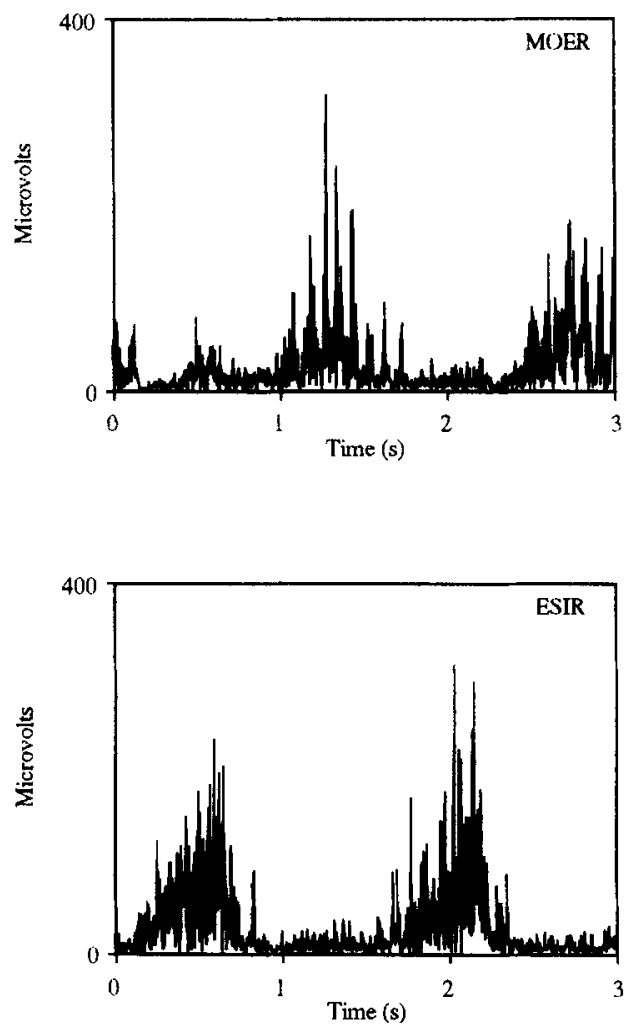

Fig. 4. Rectified MES recorded simultaneously from right-sided muscles during a cyclic attempted flexion-extension task demonstrate alternating bursts of flexor and extensor activities. The cyclic task rate was $40 \mathrm{cpm}$ and the peak exertions were $60 \%$ of maximum voluntary effort levels. 

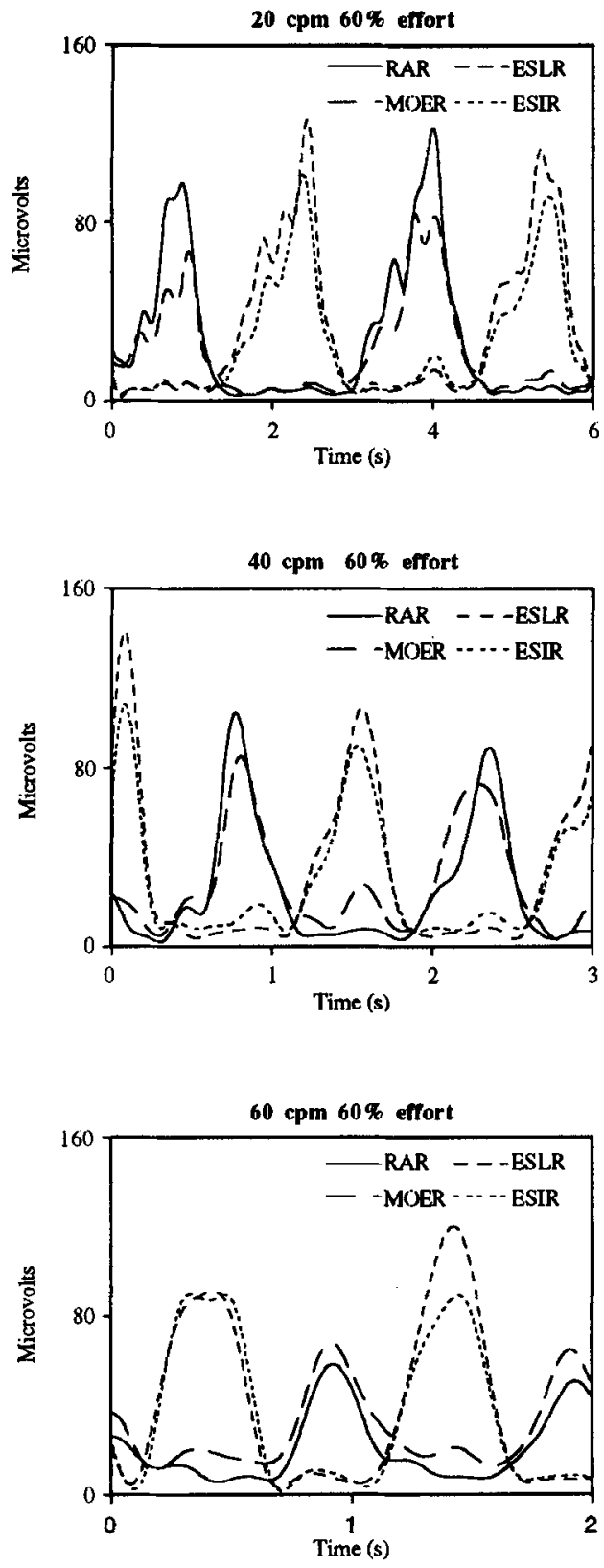

Fig. 5. Rectified and low-pass filtered MES during sample cyclic exertion tasks at rates of 20,40 and $60 \mathrm{cpm}$. Minimal overlap of flexor and extensor muscle activity is observed.

ESL, and $202 \mathrm{~ms}$ for the ESI while the mean time shifts at the $60 \mathrm{cpm}$ and $60 \%$ effort level were $119 \mathrm{~ms}$ for the RA, $118 \mathrm{~ms}$ for the MOE, $145 \mathrm{~ms}$ for ESL, and $138 \mathrm{~ms}$ for the ESI. The MES-force time shifts were found to be substantially bilaterally symmetric for each subject and, in the mean, across subjects.

Sample plots of the best linear regression through the origin between time-shifted rectified and filtered MES and the biomechanical model-predicted muscle forces demonstrate a nearly linear MES-force relationship (Fig. 8). The error between MES and biomechanical model-predicted forces, expressed as a
Table 2. Mean normalized cross-correlations between rectified and filtered MES from agonistic muscle pairs. The high correlations demonstrates a large degree of constant load sharing between agonistic muscles

\begin{tabular}{lcccc}
\hline \multicolumn{2}{c}{ Nominal } & & & \\
\cline { 1 - 2 } \cline { 5 - 5 } Rate (cpm) & Effort (\%) & & RA-MOE & ESL-ESI \\
\hline 20 & 20 & & 0.86 & 0.95 \\
40 & 20 & & 0.83 & 0.95 \\
60 & 20 & & 0.85 & 0.96 \\
20 & 40 & & 0.89 & 0.96 \\
40 & 40 & & 0.90 & 0.95 \\
60 & 40 & & 0.92 & 0.97 \\
20 & 60 & 0.94 & 0.97 \\
40 & 60 & 0.91 & 0.97 \\
60 & 60 & 0.91 & 0.96 \\
\hline
\end{tabular}

Note: See Table 1 for abbreviations.

percentage, ranged from 20 to $36 \%$ (Table 4). The MES-force errors were largest for the medial oblique muscles and smallest for the erector spinae muscles. The errors demonstrated no strong association with task rate for any of the muscles.

The isometric cyclic task MES-force gains for each muscle of each subject were normalized by the static MES-force gains for that muscle. Greater inter-subject and task-to-task variability is seen in the normalized gains of the abdominal muscles than the back muscles (Table 5). The normalized MES-force gains for the ESI decreased as the task rate and effort level were increased. The normalized gains for the ESL decreased as the task rate was increased, but increased as the effort level was increased at a fixed rate.

\section{DISCUSSION}

A linear relationship was found between mean model-predicted muscle forces and mean rectified MES over a series of statically attempted trunk flexions and extensions. This agrees with the linear mean rectified MES-force relationship previously found for the trunk muscles for low-to-moderate effort level static sagittal loadings of the trunk (Schultz et al., 1983; Seroussi and Pope, 1987). The errors between MES-predicted and biomechanical model-predicted muscles forces were on the order of $20 \%$. These errors are larger than the $10 \%$ errors between extensor MES and torque found by Stokes et al. (1987) using a linear model. However, the study by Stokes et al. (1987) did not include attempted flexion and also did not use regression through the origin as was used in this study. It is believed that these effects contribute to the larger errors found here.

Cyclic exertions of various rates and effort levels were used to investigate the quantitative relationship between MES and muscle force during rapidly varying isometric contractions. The dynamic relationship between the rectified MES from a single muscle and net 

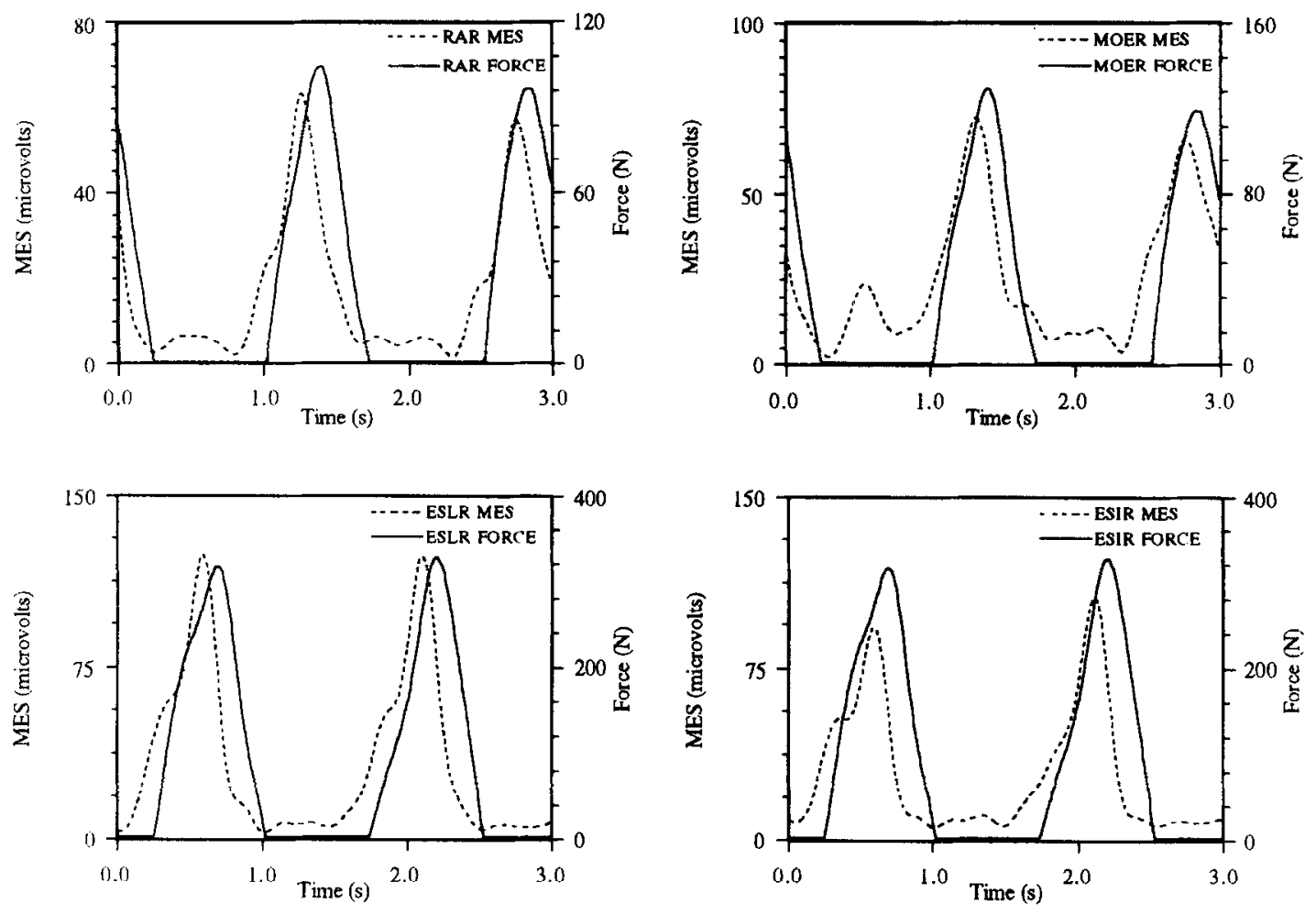

Fig. 6. Predicted lumbar muscle forces and associated rectified and low-pass-filtered MES demonstrate the substantial phase lag between MES and muscle force. The cyclic task rate was $40 \mathrm{cpm}$ and the peak exertions were $60 \%$ of maximum voluntary effort levels.
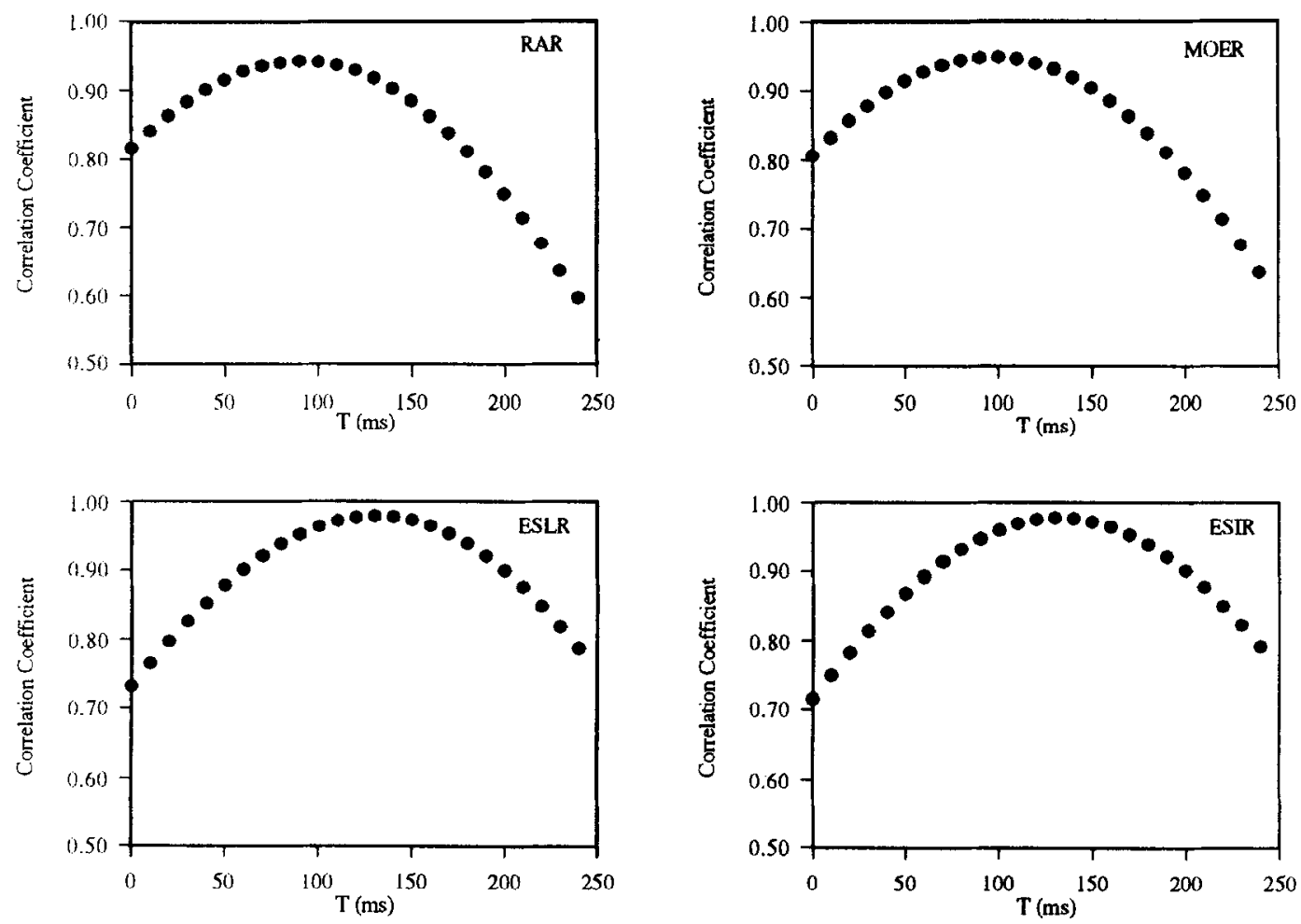

Fig. 7. Sample plots demonstrating the effect of the time shift on the linear correlation between the timeshifted, rectified and filtered myoelectric signals and associated predicted trunk muscle forces. The cyclic task rate was $40 \mathrm{cpm}$ and the peak exertions were $60 \%$ of maximum voluntary effort levels. 

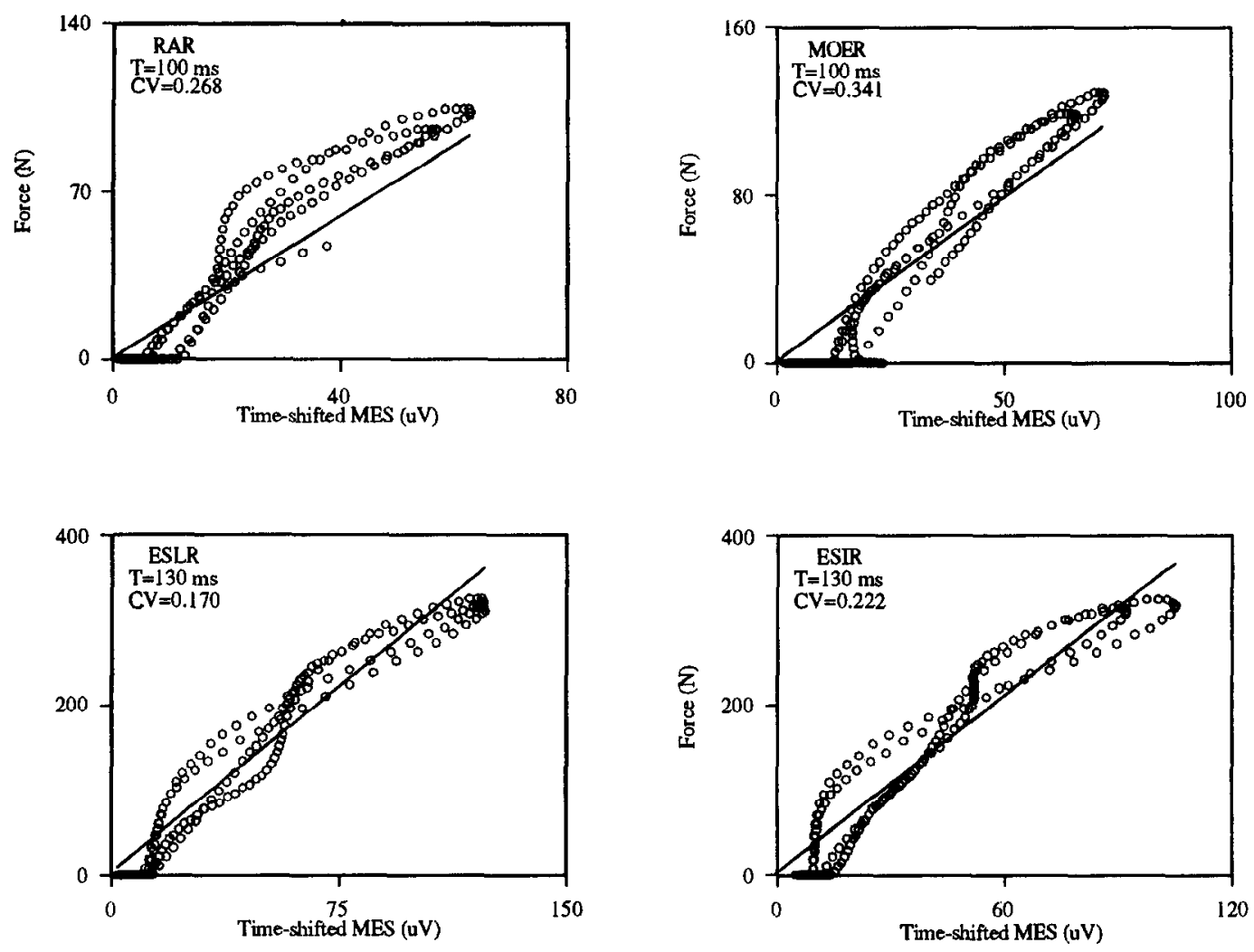

Fig. 8. Best linear regression through the origin between the predicted muscle contraction forces and the time-shifted, rectified and filtered myoelectric signals during a cyclic isometric task. The cyclic task rate was $40 \mathrm{cpm}$ and the peak effort was $60 \%$ of maximum voluntary effort.

Table 3. Mean time shifts $(T)$ that maximized the linear cross-correlation between the biomechanical model-predicted muscle forces and rectified and filtered MES for each cyclic task. The time shifts demonstrate dependence on the muscle, rate and effort level

\begin{tabular}{|c|c|c|c|c|c|c|c|c|c|}
\hline \multicolumn{2}{|c|}{ Nominal } & \multicolumn{8}{|c|}{$T(\mathrm{~ms})$} \\
\hline \multirow{2}{*}{$\begin{array}{l}\text { Rate } \\
\text { (cpm) }\end{array}$} & \multirow{2}{*}{$\begin{array}{c}\text { Effort } \\
(\%)\end{array}$} & \multicolumn{2}{|c|}{ RA } & \multicolumn{2}{|c|}{ MOE } & \multicolumn{2}{|c|}{ ESL } & \multicolumn{2}{|c|}{ ESI } \\
\hline & & Mean & SD & Mean & SD & Mean & $\mathrm{SD}$ & Mean & SD \\
\hline 20 & 20 & 202 & (18) & 180 & $(24)$ & 218 & (29) & 203 & $(37)$ \\
\hline 40 & 20 & 174 & (38) & 161 & (40) & 189 & (31) & 186 & (17) \\
\hline 60 & 20 & 172 & (25) & 177 & (25) & 166 & (25) & 172 & (16) \\
\hline 20 & 40 & 148 & (41) & 146 & (31) & 189 & (28) & 166 & (21) \\
\hline 40 & 40 & 136 & (36) & 127 & (37) & 167 & (21) & 150 & (21) \\
\hline 60 & 40 & 134 & (22) & 138 & (17) & 150 & (28) & 150 & (23) \\
\hline 20 & 60 & 139 & (18) & 132 & (30) & 189 & (25) & 163 & (27) \\
\hline 40 & 60 & 127 & (20) & 111 & (28) & 153 & (28) & 139 & (24) \\
\hline 60 & 60 & 119 & (22) & 118 & (17) & 145 & (21) & 138 & (21) \\
\hline
\end{tabular}

Note: See Table 1 for abbreviations.

time-varying joint torque has previously been described by a second-order linear differential equation (Cogshall and Bekey, 1970; Gottlieb and Agarwal, 1971; Crosby, 1978). The second-order differential equations reported are low-pass systems which are either critically or slightly overdamped. Olney and Winter (1985) estimated joint torques about the knee and ankle by low-pass filtering rectified myoelectric activities with a second-order digital filter. The filter cutoff frequency was selected for each muscle such that the correlation between rectified and filtered myoelectric activity and net torque was maximal during an isometric cyclic calibration task. Low-pass filtering accounts for the gain and phase relationship between MES and the muscle contraction force.

In the present study, rectified MES were processed using a linear low-pass digital filter with forward and backward passes so that the filter caused no net phase 
Table 4. The error between the biomechanical model-predicted and MES-predicted muscle forces expressed as the coefficient of variability for the highest effort level tasks. The errors were smallest for the erector spinae muscles and largest for the abdominal oblique muscles

\begin{tabular}{|c|c|c|c|c|c|c|c|c|c|}
\hline \multicolumn{2}{|c|}{ Nominal } & \multicolumn{8}{|c|}{$\mathrm{CV}$} \\
\hline \multirow{2}{*}{$\begin{array}{l}\text { Rate } \\
(\mathrm{cpm})\end{array}$} & \multirow{2}{*}{$\begin{array}{c}\text { Effort } \\
(\%)\end{array}$} & \multicolumn{2}{|c|}{$\mathbf{R A}$} & \multicolumn{2}{|c|}{ MOE } & \multicolumn{2}{|c|}{ ESL } & \multicolumn{2}{|c|}{ ESI } \\
\hline & & Mean & $(\mathrm{SD})$ & Mean & (SD) & Mean & (SD) & Mean & (SD) \\
\hline 20 & 60 & 0.25 & $(0.08)$ & 0.33 & $(0.08)$ & 0.22 & $(0.09)$ & 0.24 & $(0.09)$ \\
\hline 40 & 60 & 0.26 & $(0.06)$ & 0.32 & $(0.06)$ & 0.20 & $(0.05)$ & 0.22 & $(0.03)$ \\
\hline 60 & 60 & 0.28 & $(0.07)$ & 0.36 & $(0.08)$ & 0.21 & $(0.05)$ & 0.23 & $(0.03)$ \\
\hline
\end{tabular}

Note: See Table 1 for abbreviations.

Table 5. The mean normalized MES-force gains for each cyclic task. The ESI mean gains monotonically decrease with both task rate and effort level while the ESL mean gain increases with effort level at a fixed rate

\begin{tabular}{|c|c|c|c|c|c|c|c|c|c|}
\hline \multicolumn{2}{|c|}{ Nominal } & \multicolumn{8}{|c|}{$g_{\mathrm{c}} / g_{\mathrm{s}}$} \\
\hline \multirow{2}{*}{$\begin{array}{l}\text { Rate } \\
(\mathrm{cpm})\end{array}$} & \multirow{2}{*}{$\begin{array}{c}\text { Effort } \\
(\%)\end{array}$} & \multicolumn{2}{|c|}{ RA } & \multicolumn{2}{|c|}{ MOE } & \multicolumn{2}{|c|}{ ESL } & \multicolumn{2}{|c|}{ ESI } \\
\hline & & Mean & (SD) & Mean & (SD) & Mean & (SD) & Mean & (SD) \\
\hline 20 & 20 & 1.12 & $(0.57)$ & 0.74 & $(0.57)$ & 0.86 & $(0.27)$ & 1.07 & $(0.29)$ \\
\hline 40 & 20 & 0.71 & $(0.38)$ & 0.52 & $(0.38)$ & 0.74 & $(0.24)$ & 0.86 & $(0.30)$ \\
\hline 60 & 20 & 0.99 & $(0.58)$ & 0.72 & $(0.58)$ & 0.61 & $(0.16)$ & 0.71 & $(0.24)$ \\
\hline 20 & 40 & 1.00 & $(0.35)$ & 0.80 & $(0.35)$ & 0.96 & $(0.36)$ & 0.97 & $(0.32)$ \\
\hline 40 & 40 & 0.96 & $(0.33)$ & 0.79 & $(0.33)$ & 0.80 & $(0.23)$ & 0.80 & $(0.22)$ \\
\hline 60 & 40 & 0.99 & $(0.41)$ & 0.85 & $(0.41)$ & 0.68 & $(0.19)$ & 0.70 & $(0.18)$ \\
\hline 20 & 60 & 0.89 & $(0.25)$ & 0.89 & $(0.25)$ & 0.97 & $(0.41)$ & 0.84 & $(0.33)$ \\
\hline 40 & 60 & 0.92 & $(0.24)$ & 0.80 & $(0.24)$ & 0.87 & $(0.30)$ & 0.77 & $(0.27)$ \\
\hline 60 & 60 & 0.93 & $(0.34)$ & 0.89 & $(0.34)$ & 0.78 & $(0.23)$ & 0.68 & $(0.22)$ \\
\hline
\end{tabular}

Note: See Table 1 for abbreviations.

Table 6. Comparison of the phase lags predicted by the second-order linear transfer functions of other researchers at the nominal task rates demonstrates general agreement with the approximate MES-force phase angles found in this study

\begin{tabular}{|c|c|c|c|c|c|}
\hline \multirow[b]{2}{*}{$\begin{array}{l}\text { Frequency } \\
(\mathrm{Hz})\end{array}$} & \multirow{2}{*}{$\begin{array}{c}\begin{array}{c}\text { Gottlieb and } \\
\text { Agarwal } \\
(1971)\end{array} \\
\phi \\
\left(^{\circ}\right)\end{array}$} & \multirow{2}{*}{ 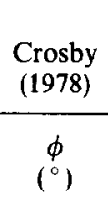 } & \multirow{2}{*}{$\begin{array}{c}\begin{array}{c}\text { Bobet and Norman } \\
(1990)\end{array} \\
\phi \\
\left({ }^{\circ}\right)\end{array}$} & \multicolumn{2}{|c|}{$\begin{array}{c}\text { Present study } \\
\text { (1992) }\end{array}$} \\
\hline & & & & $\underset{\text { (ms) }}{T}$ & $\begin{array}{c}\phi \\
\left({ }^{\circ}\right)\end{array}$ \\
\hline 0.00 & 0.0 & 0.0 & 0.0 & & \\
\hline 0.33 & 24.8 & 30.6 & 9.8 & 157 & 18.8 \\
\hline 0.67 & 47.5 & 55.6 & 20.3 & 140 & 33.6 \\
\hline 1.00 & 66.8 & 74.2 & 31.5 & 139 & 50.0 \\
\hline
\end{tabular}

shift of the myoelectric activity. In this way, the processed MES provide a direct measure of the timing of the muscular activation. Such a measure is desirable in dynamic musculoskeletal control studies where the timing of the muscular activation is important in understanding the neural control. However, pure time delays and dynamic phase shifts between MES and muscle force must be accounted for when using MES as an indicator of muscle contraction force. In the present study, the time shifts that maximized the linear correlation between rectified and filtered MES with biomechanical model-predicted muscle forces were quantified. The magnitudes of the calculated time shifts can be compared with corresponding dynamic phase shifts between MES and muscle force predicted by the second-order rectified MES-force transfer functions reported by other researchers. For this comparison, the phase angle, $\phi$, between MES and muscle force was approximated by

$$
\phi=(f \cdot T) 360^{\circ},
$$

where $f$ is the cyclic task frequency and $T$ is the optimal time shift. As shown in Table 6, the approximate MES-force phase angles found in this study are in 
general agreement with the phase angles calculated using the rectified MES-force transfer functions reported by Bobet and Norman (1990), Crosby (1978) and Gottlieb and Agarwal (1971).

A linear dynamic model of the rectified MES-force relationship (Bobet and Norman, 1990; Olney and Winter, 1985) implies that the phase between MES and muscle force is dependent on the rate of exertion but is independent of the effort level. However, the present study found that the approximate MES-force phase angles were found to decrease as the effort level was increased for a fix cyclic task rate. For example, the approximate ESI MES-force phase angle was $24^{\circ}$ during the $20 \mathrm{cpm}, 20 \%$ effort level task but decreased to $19^{\circ}$ during the $20 \mathrm{cpm}, 60 \%$ effort level task. The amplitude dependence of the MES-force phase angles is indicative of nonlinearities in the dynamic MESforce relationship. It is noted that the estimated phase lags are only approximate since the cyclic task rates used by the subject are not necessarily the nominal ones and the exertions are not perfectly sinusoidal.

It is useful to distinguish between electromechanical delay (EMD) determined by other researchers (Cavanaugh and Komi, 1979; Komi et al., 1987) and the MES-force time shift measured in the present study. While EMD is sometimes used to refer to the phase lag between MES and muscle force as calculated in the present study (Vos et al., 1990), EMD is best defined as a pure time delay between the onset of MES and the onset of muscle force. Using this definition, Komi et al. (1987) determined EMD to be of the order of $27-35 \mathrm{~ms}$ for the soleus and gastrocnemius muscles. The MES-force time shift found in this study includes EMD as well as the dynamic phase shift between MES and muscle force associated with the dynamics of an isometrically contracting muscle. The dynamic phase shift is, in general, dependent on the rate of muscular activation or deactivation during an entire time history of a contraction (Vos et al., 1990). Our methods do not allow us to distinguish what portions of the time shift are attributable to EMD and the dynamic phase shift.

The MES-force phase lags measured in this study are to some degree dependent on the biomechanical model assumption of the use of minimal antagonism. The biomechanical model predicts that no cocontraction is used when the direction of the attempted motion changes. Cocontraction of agonists and antagonists can occur when the subject switches between attempted flexion and extension and vice versa, although little cocontraction is evident in Fig. 5. If it does occur, then the effect of simultaneous flexor and extensor muscle activities would be to slightly increase the calculated MES-force phase difference.

The normalized MES-force gains displayed a high degree of variability from subject to subject for a specific cyclic task. These variabilities are partially attributable to inaccuracies in the biomechanical model assumptions and/or variability of MES measurements. An assumption inherent in the double linear programming lumbar muscle force prediction model during sagittal loading is that there is an almost equal stress distribution among agonistic moments. Vink et al. (1988) used MES measurements to study load sharing between medial, intermediate and lateral columns of the erector spinae muscle during the development of extension moments. They found that medial columns of the erector spinae were recruited greater than lateral columns at low effort levels while lateral columns were recruited greater at higher effort levels. This trend may be reflected in the calculated MES-force gains which show the ESL gain increasing with increased effort level at a fixed rate while the ESI gain decreases with increased effort level at a fixed rate.

Variability in MES measurements can also contribute to the variable gains and have been previously observed by Siegler et al. (1985) when subjects performed repeated quasi-static isometric contractions. Similarly, Stokes et al. (1987) found a nonlinear MES-torque relationship for back extensor muscles, but reported that a linear model was more repeatable due to the task-to-task variability in MES that was observed. Our experience is that carefully controlling the posture during task performances can help redace MES variability but does not completely eliminate it. A lack of repeatability in MES measurements remains a major drawback in using MES to quantify muscle loads.

Regression through the origin was used to relate the processed myoelectric activities to the predicted forces. This regression would result in poor agreement, as measured by the coefficient of variability, if there was pronounced muscle activity when zero muscle force was predicted. The greatest error using this linear model was found for the medial oblique muscle, which suggests the greatest amount of antagonism was in this muscle. Schultz et al. (1983) and Zetterberg et al. (1987) also reported weaker correlations between biomechanical model-predicted muscle forces and MES for the oblique muscles than the rectus abdominis and erector spinae muscles during static task performances. This may be partially attributable to the oblique muscle being used to stiffen the structures of the trunk during attempted extensions.

Acknowledgement-The support for this research of U.S. National Institutes of Health Grants AG 06621 and NS 20536 is gratefully acknowledged.

\section{REFERENCES}

Bean, J. C., Chafin, D. B. and Schultz, A. B. (1988) Biomechanical model calculation of muscle contraction forces: a double linear programming method. J. Biomechanics 21, 59-66.

Bobet, J. and Norman, R. W. (1990) Least-squares identification of the dynamic relation between the electromyogram and joint moment. J. Biomechanics 23, 1275-1276.

Cavanaugh, P. R. and Komi, P. V. (1979) Electromechanical delay in human skeletal muscle under concentric and 
eccentric contractions. European J. appl. Physiol. 42, 159-163.

Cogshall, J. C. and Bekey, G. A. (1970) EMG-force dynamics in human skeletal muscle. Med. Biol. Engng 8, 265-270.

Crosby, P. A. (1978) Use of surface electromyogram as a measure of dynamic force in human limb muscles. Med. Biol. Engng Comput. 16, 519-524.

Eycleshymer, A. C. and Schoemaker, D. M. (1911) A CrossSection Anatomy. Appleton-Century Crofts, New York.

Gottlieb, G. L. and Agarwal, G. C. (1971) Dynamic relationship between isometric muscle tension and the electromyogram in man. $J$. appl. Physiol. 30, 345-351.

Komi, P. V., Salonen, M., Jarvinen, M. and Kokko, O. (1987) In vivo registration of achilles tendon forces in man. $\mathbf{I}$. methodological development. Int. J. Sports Med. 8, 3-8.

McConville, J. T., Churchill, T. D., Kaleps, I., Clauser, C. E. and Cuzzi, J. (1980) Anthropometric relationships of body and body segment moments of inertia. AFAMRL-TR80-119, Aerospace Medical Research Laboratories, Wright-Patterson Air Force Base, $\mathrm{OH}$.

McGill, S. M., Norman, R. W. (1986) Partitioning of the L4-L5 dynamic moment into disc ligamentous and muscular components during lifting. Spine 11, 666-677.

Olney, S. J. and Winter, D. A. (1985) Predictions of knee and ankle moments of force in walking from EMG and kinematic data. J. Biomechanics 18, 9-20.

Schultz, A. B. (1990) Models for analyses of lumbar spine loads. Appl. Mech. Rev. 43, 119-125.

Schultz, A. B., Cromwell, R., Warwick, D. and Andersson, G. (1987) Lumbar trunk muscle use in standing isometric heavy exertions. J. Orthop. Res. 5, 320-329.

Schultz, A. B., Haderspeck, K., Warwick, D. and Portillo, D. (1983) Use of lumbar trunk muscles in isometric performance of mechanically complex standing tasks. $J$. Orthop. Res. 1, 77-91.

Seroussi, R. E., and Pope, M. H. (1987) The relationship between trunk muscle electromyography and lifting moments in the sagittal and frontal planes. J. Biomechanics 20, $135 \cdot 146$.

Siegler, S., Hilstrom, H. J., Freedman, W. and Moskowitz, G. (1985) Effect of myoelectric signal processing on the relationship between muscle force and processed EMG Am. J. Phys. Med. 64, 130-149.

Stokes, I. A. F., Moffroid, M., Rush, S. and Haugh, L. D. (1989) EMG to torque relationship in rectus abdominis muscle: results with repeated testing. Spine 14, 857-861.

Stokes, I. A. F., Rush, S., Moffroid, M., Johnson, G. B. and Haugh, L. D. (1987) Trunk extensor EMG-torque relationship. Spine 12, 770-776.

Vink, P., van der Velde, E. A. and Verbout, A. J. (1988) A functional subdivision of the lumbar extensor musculature. Electromyogr. Clin. Neurophysiol. 28, 517-525.

Vos, E. J., Mullender, M. G. and van Ingen Schenau, G. J. (1990) Electromechanical delay in the vastus lateralis muscle during dynamic isometric contractions. Europe'an J. appl. Physiol. 60, 467-471.

Zetterberg, C., Andersson, G. B. J. and Schultz, A. B. (1987) The activity of individual trunk muscles during heavy physical loading. Spine 12, 1035-1040. 